\section{Prehospital thrombolysis}

\section{Views of non-users ignored}

EDITOR,-In his assimilation of general practitioners' views on prehospital thrombolysis John Rawles fails to look in detail at the subgroup from whom we are most likely to gain an interesting insight-namely, those general practitioners who took part in the Grampian region early anistreplase trial. ${ }^{1}$ Only 25 of the 87 doctors who took part in the study (a study which he suggests shows that the efficacy of thrombolysis is doubled when it is given before admission to hospital) identified themselves as current users of thrombolysis. Why did the remaining doctors stop using this treatment?

Rawles's main conclusions are that general practitioners feel the need to be better educated and value the input of local cardiologists in establishing protocols and management guidelines. I would have thought that those general practitioners who took part in the Grampian study had been sufficiently well educated and supported by local cardiologists. Why, when these two barriers to use have been removed and the case for the effectiveness of the treatment has been well made, do large numbers of general practitioners feel unable to change their behaviour? Has it anything to do with the cost of the treatment? Given that the second international study of infarct survival suggests that aspirin is as effective as streptokinase, perhaps the role of general practice should be to ensure that this safer treatment is started as soon as possible.

It is disconcerting to find that $22 \%$ of the general practitioners who gave thrombolytic drugs felt confident of doing so without recourse to electrocardiography and that $45 \%$ were happy to give them despite having no access to a defibrillator The users also reported, on average, over $50 \%$ more cases of suspected myocardial infarction than non-users $(5.27 v 3.30)$. Assuming that this number of events is based on an estimate rather than corroborated evidence, what does this tell us about general practitioners' index of suspicion? The variations seem unlikely to reflect differences in the practice populations.

Rawles's paper raises more questions about attitudes than it answers. This would not have been the case if the general practitioners in the Grampian study alone had been more appropriately surveyed

Reading RG3 2BA

1 Rawles J. Attitudes of general practitioners to prehospital thrombolysis. $B M \mathcal{F}$ 1994;309:379-82. (6 August.)

\section{Suits rural areas}

EDITOR,-The Grampian region early anistreplase trial $^{1}$ and John Rawles's study of prehospital thrombolysis ${ }^{2}$ need to be considered in their geographical context. Rural areas where the time taken to transfer a patient to a cardiac care unit (even with a fast track system) is potentially long are ideal for this lifesaving treatment.

In my urban practice the maximum distance to the local unit is about $10 \mathrm{~km}$. I have always taken the view that, because of the short transfer time and because all ambulances in Cleveland have
We prefer short letters that relate to a recently published article and we are unlikely to publish letters longer than 400 words and containing over five references. Letters may be shortened. Your letters should be typed with double spacing and include a word count. All authors need to sign the letter and provide one current appointment and address. We encourage you to declare any conflict of interest. Please enclose a stamped addressed envelope if you require an acknowledgment.

paramedics aboard, the best care for my patients is achieved by early, quick admission to hospital, with thrombolysis being given there. Therefore I do not carry any thrombolytic drugs in my medical bag. Recent difficulties with beds locally, however, have on occasion led to delays in arranging beds for admissions, albeit not yet for patients with cardiac symptoms. Perhaps I will need to rethink my rationale and propose a new indication for thrombolysis-"lack of availability of a bed"- - and start carrying appropriate treatment.

IAIN BONAVIA

Tennant Street Surgery, General practitioner

Stockton on Tees,

Cleveland TS18 2AT

1 GREAT Group. Feasibility, safety, and efficacy of domicilliary thrombolysis by general practitioners. $B M Y$ 1992;305:548-53. 2 Rawles J. Attitudes of general practitioners to prehospital thrombolysis. $B M F$ 1994;309:379-82. (6 August.)

\section{Thrombolysis should be used in hospitals}

EDITOR,-It has been shown that early prehospital thrombolysis reduces mortality from myocardial infarction at one year, and John Rawles suggests that general practitioners should be encouraged to give thrombolytic treatment to patients before admission to hospital. ${ }^{1}$ I agree that prehospital care of patients with chest pain must be optimised, but hospitals must also address the issue of delay in the management of such patients. Most general practitioners do not wish to give thrombolytic treatment at home. ${ }^{2}$ Many have now developed mechanisms, involving practice staff at all levels, to minimise delays in attending patients with chest pain at home, and the administration of aspirin before admission is becoming routine.

In this district general practitioners can refer patients direct to the coronary care unit, and ambulance paramedical staff attending patients with suspected myocardial infarction can take them direct to the unit for assessment and treatment, avoiding the inevitable delay in the accident and emergency department. A rapid response by general practitioners and ambulance crews ensures minimal delay before admission and, in this hospital, is followed by immediate assessment and consideration for thrombolytic treatment in the coronary care unit.

Rawles's suggestion that the small number of cardiologists in Britain makes it difficult for hospital workers to give thrombolysis safely does not support the argument that general prac- titioners should be encouraged to give prehospital thrombolysis. Patients admitted to coronary care units throughout Britain are assessed and treated by experienced nursing and medical staff in units where thrombolysis can be given safely with full monitoring and support facilities.

Hospitals must maintain and follow protocols for the acute management of patients with suspected myocardial infarction and continually audit response times to ensure minimal delays to thrombolytic treatment. The role of general practitioners should be to ensure minimal delay in visiting patients at home, assessing them, and transferring those with suspected myocardial infarction to hospital; to give aspirin and opiates before admission; and to perform resuscitation when appropriate.

KIERON COONEY Paediatric registrar

St Mary's Hospital NHS Trust,

Newport,

Isle of Wight PO30 5TG

1 Rawles J. Attitudes of general practitioners to prehospital thrombolysis. BMF 1994;309:379-82. (6 August.)

2 Round A, Marshall AJ. Survey of general practitioners' prehospital management of suspected acute myocardial infarction. $B M F$ 1994;309:375-6. (6 August.)

\section{Is very expensive}

EDITOR,-I question John Rawles's assertion that general practitioners can be encouraged to use thrombolytic agents successfully in the community ${ }^{1}$ because so few cover their own patients out of hours. The Grampian region early anistreplase trial was an overwhelming success, but anistreplase is expensive at $£ 582$ (including value added tax) compared with the streptokinase preparations given by junior hospital staff, which cost under $£ 100$ a dose. ${ }^{2}$ With overwhelming pressure on general practitioners to reduce their prescribing costs it is no surprise that giving domiciliary thrombolysis has failed to become standard NHS practice, even in rural areas. I no longer use anistreplase but start a standard infusion of streptokinase with $100 \mathrm{mg}$ hydrocortisone cover. ${ }^{3}$ Slow infusions are safer than bolus injections: while they carry the same risks, they can be stopped if adverse side effects develop. As streptokinase does not require refrigerated storage it can be kept in the practice's car ready for immediate use.

Giving sets, intravenous fluids, and cannulas are not available to general practitioners in the NHS since their costs are not reimbursed by the Prescription Pricing Authority. Even if their costs are added to the cost of streptokinase ( $£ 80$ plus value added tax), however, this will still be considerably cheaper than anistreplase. Vocationally trained doctors are capable of setting up slow intravenous infusions and monitoring their patient on the journey to hospital. As long as they are appropriately equipped I see no reason why general practitioners should not start a standard streptokinase infusion, but deputies do not carry thrombolytic agents. In cases of suspected myocardial infarction and other serious illness or injury, controllers working for commercial deputising services and for general practice cooperatives routinely transfer calls to the ambulance service.

Until general practitioners are adequately rewarded for providing personal out of hours cover 